\title{
AVALIAÇÃO DE DUAS INTERVENÇÕES EDUCATIVAS PARA A PREVENÇÃO DO DIABETES MELLITUS TIPO 2 EM ADOLESCENTES
}

\author{
Ana Roberta Vilarouca da Silva ${ }^{1}$, Maria Lúcia Zanetti ${ }^{2}$, Adriana Costa e Forti ${ }^{3}$, Roberto Wagner Júnior Freire \\ de Freitas ${ }^{4}$, Miguel Nasser Hissa ${ }^{5}$, Marta Maria Coelho Damasceno ${ }^{6}$
}

\footnotetext{
${ }^{1}$ Doutora em Enfermagem. Professora do Departamento de Enfermagem da Universidade Federal do Piauí. Piauí, Brasil. E-mail: robertavilarouca@yahoo.com.br

2 Doutora em Enfermagem. Professora Associado do Departamento de Enfermagem Geral e Especializada da Escola de Enfermagem de Ribeirão Preto, da Universidade de São Paulo. São Paulo, Brasil. E-mail: zanetti@eerp.usp.br

${ }^{3}$ Doutora em Medicina. Professora do Departamento de Medicina Clínica da Universidade Federal do Ceará. Ceará, Brasil. E-mail: adrianaforti@uol.com.br

${ }^{4}$ Doutorando do Programa de Pós-Graduação em Enfermagem da Universidade Federal do Ceará. Ceará, Brasil. E-mail: rwjff@ globo.com

${ }^{5}$ Doutor em Medicina. Professor do Departamento de Medicina Clínica da Universidade Federal do Ceará. Ceará, Brasil. E-mail: miguelnh@secrel.com.br

${ }^{6}$ Doutora em Enfermagem. Professora do Departamento de Enfermagem da Universidade Federal do Ceará. Ceará, Brasil. E-mail: martadamasceno@terra.com.br
}

RESUMO: Objetivou-se avaliar duas intervenções educativas para a prevenção do Diabetes Mellitus tipo 2 em adolescentes de risco. Participaram 90 alunos de uma escola pública de Fortaleza, Ceará, Brasil, divididos em dois grupos: GA (n=45) e GB (n=45). O GA recebeu intervenção educativa individual, mediante um folder, e o GB, intervenção educativa grupal, abrangendo cinco encontros de duas horas cada um. Utilizaram-se questionários para avaliar o nível de conhecimento antes, um dia após e decorridos 60 dias das intervenções, e as mudanças de hábitos cotidianos, 60 dias após as intervenções. Os resultados evidenciaram que, para o nível de conhecimento, ambas as intervenções desenvolvidas não apresentaram diferença $(p=0,0001)$. Ao comparar os grupos A e B, após as intervenções, observou-se que a intenção para mudança dos hábitos cotidianos foi semelhante. As duas intervenções educativas podem ser utilizadas nas escolas para a prevenção do Diabetes Mellitus tipo 2 em adolescentes de risco.

DESCRITORES: Educação em saúde. Diabetes mellitus. Saúde do adolescente.

\section{EVALUATING TWO EDUCATIONAL INTERVENTIONS TO PREVENT TYPE 2 DIABETES MELLITUS AMONG ADOLESCENTS}

\begin{abstract}
This study aimed to evaluate two educational interventions to prevent type 2 Diabetes Mellitus among at-risk adolescents. Participants were 90 students from a public school in the city of Fortaleza, Ceará, Brazil, divided into two groups: GA (n=45), and GB $(n=45)$. In the GA group, a flyer was used to individually explain the educational group interventions, while the GB group received educational intervention through five meetings of two hours each. Questionnaires were used to evaluate the level of knowledge before, the day after, and 60 days after interventions as well as changes in daily habits, 60 days after interventions. Results showed no difference between both interventions $(\mathrm{p}=0.0001)$ considering the level of knowledge. A similar intention to change daily habits was observed upon comparing groups A and B after the interventions. The two educational interventions can be used in schools in order to prevent type 2 Diabetes Mellitus in at-risk adolescents.
\end{abstract}

DESCRIPTORS: Health education. Diabetes mellitus. Adolescent health.

\section{EVALUACIÓN DE DOS INTERVENCIONES EDUCATIVAS PARA LA PREVENCIÓN DE LA DIABETES MELLITUS TIPO 2 EN ADOLESCENTES}

RESUMEN: El estudio tuvo como objetivo evaluar dos intervenciones educativas para la prevención de la Diabetes Mellitus tipo 2 en adolescentes en riesgo. Participaron del estudio noventa estudiantes de una escuela pública en la ciudad de Fortaleza, Ceará, Brasil, divididos en dos grupos: GA $(n=45)$ y GB $(n=45)$. El grupo GA recibió una intervención educativa individual, por medio de un folleto, y el grupo GB, una intervención educativa en grupo que comprendió cinco sesiones de dos horas cada una. Se utilizaron cuestionarios para evaluar el nivel de conocimientos antes, al día siguiente, y 60 días después de las intervenciones; y los cambios en los hábitos diarios 60 días después del uso de las intervenciones. Los resultados mostraron que para el nivel de conocimientos, las dos intervenciones realizadas no mostraron diferencias $(\mathrm{p}=0,0001)$. Al comparar los grupos A y B después de las intervenciones se observó que la intención de cambiar los hábitos diarios fue similar. Las dos intervenciones educativas pueden ser utilizadas en las escuelas para la prevención de la Diabetes Mellitus tipo 2 en adolescentes en riesgo.

DESCRIPTORES: Educación en salud. Diabetes Mellitus. Salud del adolescente. 


\section{INTRODUÇÃO}

O Diabetes Mellitus tipo 2 (DM2) é uma doença frequente, e sua prevalência está aumentando no mundo. Estima-se que o número de indivíduos acometidos deverá sofrer um incremento de $50 \%$ até o ano de 2025..$^{1-2}$ A progressiva ascensão dessa doença crônica impõe a necessidade de uma revisão das práticas dos serviços de saúde pública, como a implementação de ações de saúde e estratégias eficazes de prevenção e controle. ${ }^{3}$

Estudo de meta-análise mostrou aumento da incidência global do DM2 em crianças e adolescentes e que a prevalência está interligada à obesidade em jovens e crianças nos EUA, Ásia, Nova Zelândia, Austrália, parte da Europa, América do Sul e Canadá. ${ }^{4}$

No Brasil, o DM2, uma doença típica da faixa etária acima de 40 anos, hoje acomete crianças e adolescentes, em virtude da obesidade determinada, em parte, pela genética e, em parte, pela ingestão de grandes quantidades de gordura e de calorias. Dessa forma, é preciso adotar hábitos saudáveis de vida, sobretudo em relação à alimentação e à atividade física, uma vez que o sedentarismo também tem sua parcela de contribuição no quadro atual. ${ }^{5}$

A magnitude do problema vem despertando cada vez mais o interesse de pesquisadores, que apontaram como foco das discussões nos eventos específicos agendados para o período entre 2009 e 2013, a educação em diabetes e a prevenção do DM2.

O Diabetes Mellitus (DM) preenche quatro das cinco condições para justificar o início de um programa de prevenção. ${ }^{6}$ Por envolver os fatores de risco mencionados anteriormente, a prevenção deve abranger, também, outras doenças, como as cardiovasculares.

Nessa direção, o Ministério da Saúde recomenda que as intervenções em saúde devam ocorrer antes da manifestação clínica do DM. Assim, são indicadas medidas preventivas, aliadas às ações assistenciais que demandem práticas de saúde mais abrangentes para a população, a fim de identificar e/ou combater os fatores de risco, ou reduzir a oportunidade de exposição das pessoas. ${ }^{\text {? }}$

Estudos mostram a importância da identificação de fatores de risco para DM2 em adolescentes. ${ }^{8-9}$ No entanto, constatou-se, após busca aprofundada em bases de dados, a escassez da literatura sobre intervenções educativas efetivas na prevenção do DM2.
Ao considerar que inquéritos anteriores sobre os fatores de risco para DM2 em adolescentes de Fortaleza-CE ${ }^{8-9}$ mostraram alto percentual de excesso de peso e sedentarismo, e que há necessidade de testar intervenções educativas para prevenir essa doença, o presente estudo teve como objetivo de avaliar duas intervenções educativas para a prevenção do DM2 em adolescentes com fatores de risco.

\section{MÉTODO}

Estudo quase-experimental, comparativo, prospectivo, realizado de janeiro de 2007 a novembro de 2009. Participaram adolescentes de ambos os sexos, com idade entre 14 e 19 anos, matriculados em uma escola pública da rede estadual de ensino, da cidade de Fortaleza-CE. Os sujeitos foram selecionados por meio de consulta ao banco de dados, construído a partir da investigação sobre os fatores de risco para DM2, em alunos de escolas públicas de Fortaleza-CE. ${ }^{8}$

Os critérios de inclusão estabelecidos foram: ter pelo menos um dos fatores de risco modificáveis para DM2 (excesso de peso, sedentarismo, glicemia capilar e/ou valores da pressão arterial elevado), e estar frequentando a escola no período da coleta de dados. Excluíram-se os casos confirmados de DM e os adolescentes com deficiência cognitiva e/ou de leitura ou de escrita.

Para o cálculo amostral utilizou-se a fórmula adaptada para situações que, a priori, envolvem dois grupos predefinidos. ${ }^{10} \mathrm{~A}$ amostra foi constituída por 90 adolescentes, os quais foram subdivididos em dois grupos designados como GA e GB, cada um com 45 participantes, sendo que o GA recebeu intervenção educativa individual e o GB, em grupo.

A alocação nos grupos ocorreu de forma aleatória e os estudantes não sabiam a qual grupo pertenciam. Foi utilizado um formulário para o registro das variáveis sociodemográficas (sexo, idade, série e renda familiar) e clínicas (índice de massa corporal (IMC) específico para adolescentes, ${ }^{11}$ atividade física, glicemia capilar ao acaso e pressão arterial), além de questionários para avaliar o nível de conhecimento e as mudanças de hábitos cotidianos antes e após as intervenções educativas.

Em relação à pratica de atividade física foi considerado sedentário o adolescente que realizava exercícios físicos por um tempo inferior a trinta minutos e com uma frequência menor do que três vezes por semana. ${ }^{12} \mathrm{~A}$ glicemia capilar ao acaso foi classificada segundo as recomendações da Socie- 
dade Brasileira de Diabetes ${ }^{13}$ e a pressão arterial, de acordo os valores preconizados pela The Fourth Report on the Diagnosis, Evaluation, and Treatment of High Blood Pressure in Children and Adolescents. ${ }^{14}$ O nível de conhecimento, antes e após as intervenções, foi classificado em seis categorias. ${ }^{15}$ No entanto, para alocar os resultados obtidos nas categorias atribuiu-se intervalos de notas de zero a 10 como segue: nenhum conhecimento (zero), muito pouco $(<1-2,9)$, pouco $(3-4,9)$, bom $(5-6,9)$, mais que bom $(7-8,9)$, muito bom (9-10). No que diz respeito às mudanças de hábitos foi considerada a prontidão (intenção de mudança) dos adolescentes para modificar a alimentação e introduzir a prática de atividade física no cotidiano.

As intervenções educativas, individual e em grupo, foram realizadas na escola, obedecendo quatro fases: 1 - avaliação do nível de conhecimento sobre o DM2, os fatores de risco e as formas de prevenção, nos grupos GA e GB; 2 - início das intervenções educativas; 3 - avaliação do nível de conhecimento sobre o DM2, os fatores de risco e as formas de prevenção, nos grupos GA e GB em duas ocasiões distintas: no dia seguinte à intervenção e decorridos 60 dias; e 4 - avaliação da prontidão para mudanças nos hábitos cotidianos, passados 60 dias do término das intervenções educativas. Os pesquisadores estabeleceram esse prazo em função do tempo estimado para o desenvolvimento do estudo e da duração do semestre escolar dos alunos.

A intervenção educativa individual incluiu a montagem, na escola, de uma espécie de consultório, onde os alunos, individualmente, receberam um folder com informações sobre DM2 (conceito, sinais e sintomas, complicações agudas e crônicas, fatores de risco e formas de prevenção) e, em seguida, foram dadas, pela pesquisadora, explicações breves sobre esse conteúdo. Foram marcados encontros para cada dez alunos, uma vez por semana, até completar as intervenções com os participantes do GA.

Na intervenção educativa em grupo aplicou-se um programa de curta duração que privilegiou a educação participativa, as crenças, as opiniões e as necessidades de aprendizagem, bem como a interação pesquisadora-aluno. Realizaram-se encontros semanais desenvolvidos nos seguintes moldes: o grupo foi subdividido em duas turmas, uma com 23 e outra com 22 alunos. Ambas receberam a intervenção educativa na mesma semana. O programa teve carga horária total de dez horas-aula, distribuídas em cinco encontros semanais, com duas horas de duração cada um, e abrangeu conteúdo idêntico ao recebido pelo GA.

Como o objetivo foi avaliar duas intervenções educativas para a prevenção do DM2 em adolescentes com fatores de risco, a análise dos dados envolveu a comparação dos resultados sobre o nível do conhecimento antes e após as intervenções, tanto para o GA quanto para o GB, e entre os dois grupos. O mesmo procedimento foi realizado para os dados obtidos sobre as mudanças nos hábitos cotidianos.

Analisou-se a confiabilidade dos dados, utilizando-se o coeficiente de correlação intraclasse. Para comparar o nível de conhecimento antes e após as intervenções educativas utilizaram-se os testes paramétricos $\mathrm{t}$ de Student para dados emparelhados e o teste de Fisher-Freeman-Halton. A comparação das médias das notas obtidas foi realizada pelo teste F de Snedecor. Para a análise de associação utilizaram-se os testes não-paramétri$\cos$ de $\chi 2$, e de razão de máximo verossimilhança. Foram consideradas estatisticamente significantes aquelas análises inferenciais cujo valor de $\mathrm{p}<0,05$.

O projeto foi aprovado pelo Comitê de Ética e Pesquisa em Seres Humanos da Universidade Federal do Ceará, sob protocolo 260/07. Todos os sujeitos assinaram o Termo de Consentimento Livre e Esclarecido e os menores de dezoito anos tiveram os termos também assinados pelos pais e/ou responsáveis.

\section{RESULTADOS}

\section{Caracterização dos sujeitos}

Dos 90 (100\%) sujeitos, obteve-se que $67,8 \%$ eram mulheres, porém em cada grupo havia proporções aproximadas de estudantes de ambos os sexos. No concernente à idade, $45,5 \%$ tinham de 14 a 15 anos, 41,1\%, de 16 a17 anos e 13,4\%, de 18 a 19 anos, com idade média de 15,7 anos, desvio padrão de 1,4, mediana de 16 anos e Intervalo de Confiança (IC) de 15,5 a 16,1. A distribuição da amostra por série mostrou que $35,5 \%$ estavam cursando a nona série do ensino fundamental, e $12,2 \%$, o terceiro ano do ensino médio. Aproximadamente $45,5 \%$ referiram ter renda familiar entre um e dois salários mínimos, sendo a renda média por pessoa de dois salários mínimos, com desvio padrão de 1,0, mediana de 2,0 e IC de 1,9 a 2,3.

Em relação ao nível de conhecimento, os resultados mostraram que tanto para o GA quanto para o GB houve diferença entre as avaliações realizadas antes das intervenções, um dia e 60 
dias após o termino das intervenções $(p=0,0001)$. Por sua vez, em ambos os grupos, as médias das notas obtidas um dia e 60 dias após o término das intervenções tiveram valores superiores à média da avaliação realizada antes do início das intervenções $(p=0,0001)$ (Tabela 1$)$.

Tabela 1 - Distribuição dos adolescentes dos GA e GB segundo o nível de conhecimento antes e após as intervenções. Fortaleza-CE, 2009

\begin{tabular}{|c|c|c|c|c|c|c|c|}
\hline \multirow{2}{*}{ Grupo (GA) } & \multicolumn{2}{|c|}{ Antes } & \multicolumn{2}{|c|}{ Após 1 dia } & \multicolumn{2}{|c|}{ Após 60 dias } & \multirow[b]{2}{*}{$\mathrm{p}$} \\
\hline & $\mathrm{n}$ & $\%$ & $\mathrm{n}$ & $\%$ & $\mathrm{n}$ & $\%$ & \\
\hline Nível de conhecimento & & & & & & & $0,0001^{*}$ \\
\hline Muito pouco & 1 & 2,2 & - & - & - & - & \\
\hline Pouco & 10 & 22,2 & - & - & 3 & 6,7 & \\
\hline Bom & 22 & 48,9 & 9 & 20,0 & 10 & 22,2 & \\
\hline Mais que bom & 12 & 26,7 & 26 & 57,8 & 30 & 66,7 & \\
\hline Muito bom & - & - & 10 & 22,2 & 2 & 4,4 & \\
\hline Média $^{(3)}$ & 5,4 & & 7,8 & & 7,0 & & $0,0001 \dagger$ \\
\hline DP & 1,6 & & 1,2 & & 1,2 & & \\
\hline \multicolumn{8}{|l|}{ Grupo (GB) } \\
\hline Nível de conhecimento & & & & & & & $0,0001^{*}$ \\
\hline Pouco & 7 & 15,5 & 1 & 2,2 & - & & \\
\hline Bom & 26 & 57,8 & 9 & 20,0 & 14 & 31,1 & \\
\hline Mais que bom & 12 & 26,7 & 29 & 64,4 & 25 & 55,5 & \\
\hline Muito bom & - & - & 6 & 13,4 & 6 & 13,4 & \\
\hline Média ${ }^{\ddagger}$ & 5,9 & & 7,5 & & 7,2 & & $0,0001 \dagger$ \\
\hline DP & 1,4 & & 1,2 & & 1,2 & & \\
\hline
\end{tabular}

* Teste de Fisher-Freeman-Halton para comparação das proporções de antes, após 1 dia e após 60 dias; ${ }^{\dagger}$ Teste F de Snedecor para comparação das três médias; ${ }^{\ddagger}$ Média das notas de zero a dez.

Ao se comparar as médias do nível de conhecimento dos dois grupos para as três avaliações: antes $(\mathrm{p}=0,119, \mathrm{t}=1,57)$; após 1 dia $(\mathrm{p}=0,224, \mathrm{t}=1,17)$ e após sessenta dias da intervenção $(p=0,445$, $\mathrm{t}=0,76)$, não houve diferença estatisticamente significante (Tabela 2).

Tabela 2 - Comparação das médias dos grupos em relação ao nível de conhecimento antes, após 1 dia e após 60 das intervenções educativas. Fortaleza-CE, 2009

\begin{tabular}{lcccc}
\hline \multicolumn{1}{c}{ Grupos } & Média & DP & $\mathbf{T}^{*}$ & $\mathbf{P}$ \\
\hline Antes & & & 1,57 & 0,119 \\
A & 5,4 & 1,6 & & \\
B & 5,9 & 1,4 & & \\
Após 1 dia & & & 1,17 & 0,244 \\
A & 7,8 & 1,2 & & \\
B & 7,5 & 1,2 & & \\
Após 60 dias & & & 0,76 & 0,445 \\
A & 7,0 & 1,2 & & \\
B & 7,2 & 1,2 & & \\
*Teste das médias. & & & &
\end{tabular}

Ao comparar o GA e GB, verificou-se que tanto a intervenção educativa individual quanto a grupal mostraram-se eficazes em relação à prontidão para mudança nos hábitos alimentares $(\mathrm{p}=0,371) \mathrm{e}$ na prática de atividade física $(\mathrm{p}=0,138)$. Porém sem significância estatística (Tabela 3). É importante acrescentar que os adolescentes do GA e do GB que não conseguiram mudar os hábitos alimentares e introduzir a prática de atividade física no cotidiano alegou falta de interesse e/ou que as estratégias utilizadas foram insuficientes para motivá-los.

Tabela 3 - Distribuição das respostas dos alunos de uma escola pública da rede de ensino estadual, em relação mudanças de hábitos cotidianos para a prevenção do DM2. Fortaleza-CE, 2009

\begin{tabular}{lccccc}
\hline \multirow{2}{*}{ Variáveis } & \multicolumn{2}{c}{ GA } & \multicolumn{2}{c}{ GB } & \multirow{2}{*}{$\mathbf{P}$} \\
\cline { 2 - 5 } & $\mathbf{n}$ & $\mathbf{\%}$ & $\mathbf{n}$ & $\%$ & \\
\hline Alimentação & 14 & 31,1 & 18 & 40,0 & $0,371^{*}$ \\
Sim & 31 & 68,9 & 27 & 60,0 & \\
Não & & & & & $0,138^{*}$ \\
Atividade física & 21 & 46,7 & 28 & 62,2 & \\
Sim & 24 & 53,3 & 17 & 37,8 & \\
Não & & & & & \\
\hline * Teste c ${ }^{2}$. & & & & &
\end{tabular}


É importante acrescentar que os adolescentes do GA e do GB que não conseguiram mudar os hábitos alimentares e introduzir a prática de atividade física no cotidiano referiu falta de interesse e/ ou que as estratégias utilizadas foram insuficientes para motivá-los.

\section{DISCUSSÃO}

Os resultados obtidos evidenciaram que tanto as intervenções educativas individual quanto a grupal mostraram-se eficazes para aumentar o nível de conhecimento dos adolescentes sobre o DM2, os fatores de risco e as formas de prevenção.

Esses achados estão em concordância com outros estudos que mostram, de modo geral, que as intervenções educativas individual e em grupo são eficazes tanto para a prevenção quanto para o tratamento de pessoas com DM e obesidade. ${ }^{16-21}$

A Associação Americana de Diabetes recomenda intervenções educativas estruturadas, independente da modalidade pela qual são conduzidas, que enfatizem a perda de peso moderada, incluindo a redução de calorias e menor ingestão de gordura no plano alimentar e prática regular de atividade física. ${ }^{22}$ Essas intervenções podem aumentar o nível de conhecimento dos alunos sobre os fatores de risco para o desenvolvimento do DM2.

Sabe-se que o conhecimento pode ajudar os indivíduos a modificarem o seu comportamento, a terem autonomia, a coparticiparem de decisões e atitudes relativas à sua saúde e, ainda, a serem um agente transformador de si próprio e dos outros. Por meio do desenvolvimento das competências e da aquisição de conhecimento se fortalece a habilidade e a capacitação do indivíduo (empowerment), expressão definida como o processo pelo qual as pessoas estão em situações que podem alterar o efeito da percepção de controle sobre a própria escolha.

No entanto, a aquisição de conhecimento nem sempre remete às mudanças de comportamento, pois para tanto são necessárias condições essenciais que independem da vontade das pessoas. Nesse contexto, destacam-se os fatores econômicos, sociais, culturais e motivacionais dos indivíduos e da comunidade e a implementação de políticas públicas voltadas para a promoção da saúde. Em se tratando de adolescentes, é importante considerar as especificidades dessa fase do desenvolvimento e que estão em processo de construção de uma consciência crítica sobre as questões que envolvem a saúde e a prevenção de doenças. É possível que o exposto justifique, em parte, os achados relativos à prontidão dos adolescentes para mudarem os hábitos cotidianos.

\section{CONCLUSÕES}

Ao considerar que os adolescentes investigados já tinham pelo menos um fator de risco para o desenvolvimento do DM2 conclui-se que é urgente que a escola, em parceria com as instituições de saúde, elabore intervenções que possibilitem a participação efetiva dos adolescentes nas iniciativas referentes à prevenção da doença. Também, a adoção de políticas para a modificação da alimentação oferecida nas cantinas ou pela merenda escolar poderá contribuir para a redução dos fatores de risco. Ao instituir diretrizes para a promoção da saúde na escola investigada os gestores podem escolher a intervenção educativa individual ou grupal, considerando a exequibilidade e a relação custo-benefício.

A escassez de literatura sobre as intervenções educativas para a prevenção do DM2 em adolescentes de risco constituiu uma limitação para o aprofundamento da discussão dos resultados da presente investigação. Por outro lado, indica que estudos futuros sejam desenvolvidos com essa população na busca de evidências fortes que permitam direcionar a prática no que diz à adoção de hábitos cotidianos que podem impedir ou postergar o aparecimento do DM2.

\section{REFERÊNCIAS}

1. Boyle JP, Honeycutt AA, Narayan KMV, Hoerger TJ, Geiss LS, Chen H, et al. Projection of diabetes burden through 2050: impact of changing demography and disease prevalence in the U.S. Diabetes Care. 2001 Nov; 24(11):1936-40.

2. Ministério da Saúde (BR), Organização PanAmericana da Saúde. Avaliação do plano de reorganização da hipertensão arterial e o diabetes mellitus no Brasil. Brasília (DF): MS; 2004.

3. Portero KCC, Motta DG, Campino ACC. Abordagem econômica e fluxograma do atendimento a pessoas com diabetes mellitus tipo 2 na rede pública de saúde de um município paulista. Saúde Rev. 2003 Set-Dez; 5(11):35-42.

4. Hamiel OP, Zeitler P. The global spread of type 2 diabetes mellitus in children and adolescents. J Pediatr. 2005 May; 146(5):693-700.

5. Modesto Filho J. Aumento da prevalência do DM 2 no jovem. Arq Bras Endocrinol Metab. 2003 Out; 47(Suppl):S419. 
6. American Diabetes Association. Prevenção ou retardo do diabetes do tipo 2. Diabetes Care. 2004; 3(3):119-26.

7. Ministério da Saúde (BR). Doenças crônicodegenerativas: evolução e tendências atuais. Brasília (DF): MS; 1988

8. Silva ARV, Damasceno MMC, Carvalho ZMF, Hissa MN, Almeida PC, Silva LF. Prevalencia de factores de riesgo para diabetes mellitus tipo 2 en adolescentes de Fortaleza-Brasil. Enfermería Integral. 2007 Jun; 78: 11-4.

9. Vasconcelos HCA, Araújo MFM, Damasceno MMC, Almeida PC, Freitas RWJF. Fatores de risco para diabetes mellitus tipo 2 entre adolescentes. Rev Esc Enferm USP. 2010 Dez; 44(4):881-7.

10. Pocock SJ. Clinical trials - a practical approach. Great Britiain: John Wiley \& Sons, 1989.

11. Cole TJ, Bellizzi MC, Flegal KM, Dietz WH. Establishing a standard definition for child overweight and obesity worldwide: international survey. BMJ. 2000 May; 320 (7244):1240-3.

12. Souza LJ, Neto CG, Chalita FEB, Reis AFF, Bastos DA, Filho JTDS, et al. Prevalência de obesidade e fatores de risco cardiovascular em Campos, Rio de Janeiro. Arq Bras Endocrinol Metab. 2003 Dez; 47(6):669-76.

13. Sociedade Brasileira de Diabetes. Diretrizes da Sociedade Brasileira de Diabetes; 2009.

14. National High Blood Pressure Education Program Working Group on High Blood Pressure in Children and Adolescents. The fourth report on the diagnosis, evaluation, and treatment of high blood pressure in children and adolescents. Pediatrics. 2004 Aug; 114(2 Suppl 4th Report):555-76.

15. Zernike W, Henderson A. Evaluating the effectiveness of two teaching strategies for patients diagnosed with hypertension. J Clin Nurs. 1998 Jan; 7(1):37-44.

16. Rodrigues EM, Boog MCF. Problematização como estratégia de educação nutricional com adolescentes obesos. Cad Saúde Pública. 2006 Mai; 22(5):923-31.

17. Peterson KA, Hughes M. Readiness to change and clinical success in a diabetes educational program. JABFP. 2002 Jul-Aug; 15(4):266-71.

18. Mello ED, Luft VC, Meyer F. Obesidade infantil: como podemos ser eficazes? J Pediatr. 2004 Mai-Jun; 80(3):173-82.

19. Rickheim PL, Weaver TW, Flader JL, Kendall DM Assessment of group versus individual diabetes education. Diabetes Care. 2002 Feb; 25(2):269-74.

20. Torres HC, Franco LJ, Stradioto MA, Hortale VA, Schall VT. Avaliação estratégica de educação em grupo e individual no programa educativo em diabetes. Rev Saúde Pública. 2009 Abr; 43(2):291-8.

21. Gucciardi E, Demelo M, Lee RN, Grace SL. Assessment of two culturally competent diabetes education methods: individual versus individual plus group education in canadian portuguese adults with type 2 diabetes. Ethn Health. 2007 Apr; 12(2):163-87.

22. American Diabetes Association. Clinical practice recommendations 2011. Diabetes Care. 2011 Jan; 34(Suppl 1):S1-2. 